\title{
Galectin-1 Is an Interactive Protein of Selenoprotein $M$ in the Brain
}

\author{
Xifeng Qiao ${ }^{1}$, Jing Tian ${ }^{2}$, Ping Chen ${ }^{1}$, Chao Wang ${ }^{3,4}$, Jiazuan Ni ${ }^{2}$ and Qiong Liu ${ }^{1, *}$ \\ 1 College of Life Sciences, Shenzhen Key Laboratory of Microbial Genetic Engineering, \\ Shenzhen University, Shenzhen 518060, China; E-Mails: qiaoxifeng.787@163.com (X.Q.); \\ chenpingszu@126.com (P.C.)
}

2 Department of Marine Biology, Shenzhen Key Laboratory of Marine Biotechnology and Ecology, Shenzhen University, Shenzhen 518060, China; E-Mails: jing.tianjingtj@gmail.com (J.T.); jzni@szu.edu.cn (J.N.)

3 Changchun Institute of Applied Chemistry, Chinese Academy of Sciences, Changchun 130022, China; E-Mail: raulw2003@163.com

4 University of Chinese Academy of Sciences, Beijing 100049, China

* Author to whom correspondence should be addressed; E-Mail: liuqiong@szu.edu.cn; Tel.: +86-755-2653-5432; Fax: +86-755-2653-4274.

Received: 4 September 2013; in revised form: 24 October 2013 / Accepted: 24 October 2013 / Published: 11 November 2013

\begin{abstract}
Selenium, an essential trace element for human health, mainly exerts its biological function through selenoproteins. Selenoprotein M (SelM) is one of the highly expressed selenoproteins in the brain, but its biological effect and molecular mechanism remain unclear. Thus, the interactive protein of SelM was investigated in this paper to guide further study. In order to avoid protein translational stop, the selenocysteine-encoding UGA inside the open reading frame of SelM was site-directly changed to the cysteine-encoding UGC to generate the SelM' mutant. Meanwhile, its $N$ terminal transmembrane signal peptide was also cut off. This truncated SelM' was used to screen a human fetal brain cDNA library by the yeast two-hybrid system. A new interactive protein of SelM' was found to be galectin-1 (Gal-1). This protein-protein interaction was further verified by the results of fluorescence resonance energy transfer techniques, glutathione $S$-transferase pull-down and co-immunoprecipitation assays. As Gal-1 plays important roles in preventing neurodegeneration and promoting neuroprotection in the brain, the interaction between SelM' and Gal-1 displays a new direction for studying the biological function of SelM in the human brain.
\end{abstract}


Keywords: selenoprotein M (SelM); galectin-1 (Gal-1); protein-protein interaction; yeast two-hybridization; fluorescence resonance energy transfer (FRET); co-immunoprecipitation; Glutathione $S$-transferase pull-down (GST pull-down)

\section{Introduction}

Selenium ( $\mathrm{Se}$ ) is an essential trace element for humans and animals. Se deficiency has been found in some regions in the world. Se supplementation is required for people in those areas to prevent various types of diseases, such as man reproduction, cancer, and neurodegenerative diseases [1-6]. The biological effect of selenium in human health is mainly executed via selenoproteins in which Se presents in the form of selenocysteine ( $\mathrm{Sec}$ ), the 21 st essential amino acid. This Sec residue is located in the active-site of selenoproteins and encoded by the traditional stop codon UGA in its open reading frame (ORF) of mRNA [7]. Previous studies revealed 25 selenoproteins in human and 24 selenoproteins in mouse [8]. SelM was one of those identified via bioinformatics analysis by Gladyshev in 2002 [9,10], and found to be highly expressed in the brain. However, its biological function has not been well studied, especially in the brain.

SelM has 145 amino acids, comprising an endoplasmic reticulum (ER)/Golgi-directing signal peptide in the $\mathrm{N}$-terminal, a thioredoxin-like domain in the middle, and an ER retention signal tetrapeptide (H/R/K-X-DL) in the $C$-terminal $[10,11]$. The Sec residue of SelM is located in the 48th position and present in a CXXU (i.e., Cys-Gly-Gly-Sec) motif upstream of an $\alpha$-helix. This type of CXXU motif is frequently regarded as a redox center [10]. It has been reported that the activity of antioxidant enzymes in the SelM-transgenic mice is higher than the untransgenic mice with Se treatment [12]. The treatment of Se and overexpression of SelM activated the ERK/MAPK pathway, but not the MAPK pathway involving $\mathrm{p} 38$ and JNK. It reduced the production of $A \beta_{1-42}$ by down-regulating the activity of $\beta-/ \gamma$-secretase and up-regulating the activity of $\alpha$-secretase [13]. In mouse HT22 hippocampus cells and C8-D1A cerebellum cells, SelM prevented the oxidative damage induced by hydrogen peroxide [14]. SelM knock-down in HT22 cells and primary cortical cells caused cell viability to decrease and reactive oxygen species (ROS) to increase [14]. Those reports show that SelM is an antioxidant protein possibly with neuroprotection.

In order to investigate the biological function of SelM in the brain, the interactive protein of SelM was screened by the yeast two-hybrid system and verified by the fluorescence resonance energy transfer (FRET) technique, followed by GST pull-down and coimmunoprecipitation (Co-IP) assays. Galectin-1 (Gal-1) was finally determined to be an interactive protein of SelM.

\section{Results}

\subsection{Identification of Gal-1 as an Interactive Protein of SelM'}

Selenoproteins are not expressed in yeast. In order to use the Yeast Two-hybrid System to screen the interacting protein of SelM, human SelM gene was site-directly mutated to SelM', where the Sec-coding TGA in SelM was changed to Cys-coding TGC in SelM'. Cys is an amino acid that has high 
similarity with Sec in biochemical properties. SelM' was then inserted into the vector NpGBKT7 to get the plasmid NpGBKT7-SelM'. Before library screening, the plasmid pGBKT7-SelM' and the empty plasmid pGADT7 were cotransformed firstly into AH109 yeast cells for the detection of self-activation, using the lithium acetate method. No self-activation and toxicity were observed when SelM' was expressed in the yeast cells (figures not shown). Then, the human fetal brain cDNA library was screened by $S e l M^{\prime}$ via yeast transformation and plate selection. Fourteen clones were grown and displayed blue color on the selection plates $\mathrm{SD} /$-Trp/-Leu/-His/-Ade/X- $\alpha$-gal (Figure 1B), while 13 clones displayed blue in the filter paper using the X-gal assay (Figure 1A). Among them, 12 clones simultaneously turned blue in both the $\mathrm{SD} /$-Trp/-Leu/-His/-Ade/X- $\alpha$-gal plate selection and $\mathrm{X}$-gal assay. These 12 DNA fragments originated from the fetal brain cDNA library were respectively amplified, isolated, and retransformed with the bait plasmid NpGBKT7-SelM' into Y2HGold yeast cells, followed by the plate selection (SD/-Trp/-Leu/-His/-Ade/X-a-gal). Only one blue colony (marked by a red circle and named 11 in Figures 1A,B) was displayed in the retransformation experiments, whose plasmid from the cDNA library was named pGACT2-11 (Figure 1C(a)). Meanwhile, the yeast cells cotransformed with plasmids pGACT2-11 and pGBKT7 (empty vector) did not display blue (Figure 1C(b)). Yeast cells transformed with plasmid pGACT2-11 were also streaked on the same plates, but they did not display blue, suggesting no autoactivation phenomenon for its protein expression (Figure 1C(c)). Cells co-transfected with plasmids pGBKT7-53 and pGADT7-T were used as positive control for interacting proteins (Figure 1D(b)), while those cotransfected with pGBKT7-53 and pGBKT7-Lam were used as negative control (Figure 1D(a)). The selected positive clones (No. 11) was DNA sequenced and bioinformatically analyzed to be Gal-1.

\subsection{Verification of the Protein Interaction by FRET Method}

In order to investigate whether SelM' interacts with Gal-1 in living cells, the acceptor photobleaching method was used to measure the fluorescence resonance energy transfer (FRET) between the interactive proteins. The coding sequences of SelM' and Gal-1 genes were inserted into the expression vectors containing the enhanced cyan fluorescence protein (CFP) and yellow fluorescence protein (YFP), respectively. Hela cells were cotransfected by the plasmids pECFP-C1-SelM' and pEYFP-C1-Gal-1. The cells cotransfected by the vacant plasmids pECFP-C1 and pEYFP-C1 were used as negative controls.

Those cells showing both cyan and yellow fluorescence were chosen and imaged through CFP and YFP channels. To perform receptor (YFP or YFP-Gal-1) photobleaching, the region of interest (ROI) of cells was bleached at $515 \mathrm{~nm}$ for $20 \mathrm{~s}$ in YFP channels. The cyan fluorescence of donor (CFP or CFP-SelM') increased significantly after receptor bleaching in the cells expressing CFP-SelM' and YFP-Gal-1 (Figure 2B(a,b)), but not in the negative control cells expressing CFP and YFP (Figure 2A(a,b)). As shown in Figure 2B(f), the photobleaching curve of cells expressing CFP-SelM' and YFP-Gal-1 displayed significant increase in donor fluorescence (solid curve) when receptor fluorescence decreased (dashed curve). In contrast, donor fluorescence did not significantly increase in the negative control cells (Figure 2A(f)). The FRET efficiency and the distance between donor and receptor were measured to be $27.3 \%$ and $6.35 \mathrm{~nm}$ respectively for the cells expressing CFP-SelM' and YFP-Gal-1, while those for the negative control cells were $3.2 \%$ and $8.99 \mathrm{~nm}$ respectively. The low 
FRET efficiency of control cells arose from experimental background. Those FRET results verified the interaction between SelM' and Gal-1 inside Hela cells.

Figure 1. Yeast two-hybrid screening of the human fetal brain cDNA library using Selenoprotein M (SelM') as a bait gene. (A) The yeast colonies on the plate $\mathrm{SD} /$-Ade/-Trp/-Leu/-His were picked up and streaked respectively on the squares of filter paper for X-gal display after 8-h incubation. Blue colonies indicated the interaction between prey and bait proteins; (B) The yeast colonies were streaked onto the selection plate SD/-Ade/-Trp/-Leu/-His/X- $\alpha$-gal to check for the interaction between prey and bait proteins (blue colonies); (C) Re-transformation experiments. (a) the prey plasmid pGACT2-11 and the bait plasmid NpGBKT7-SelM' were cotransformed into Y2HGold yeast cells and selected on the plate SD/-Trp/-Leu/-His/-Ade/X- $\alpha$-gal; (b) the prey plasmid pGACT2-11 and empty vector pGBKT7 were cotransformed into the yeast and selected on the plate SD/-Trp/-Leu/-His/-Ade/X- $\alpha$-gal; (c) yeast cells transformed with pGACT2-11 plasmid were restreaked on the plate $\mathrm{SD} /$-Trp/-Leu/-His/-Ade/X- $\alpha$-gal to check autoactivation of the prey protein; (D) Controls for interacting proteins. (a) yeasts cotransformed pGBKT7-53 and PGBKT7-Lam as a negative control; (b) yeasts cotransformed pGBKT7-53 and pGADT7-T as a positive control (blue color).

A

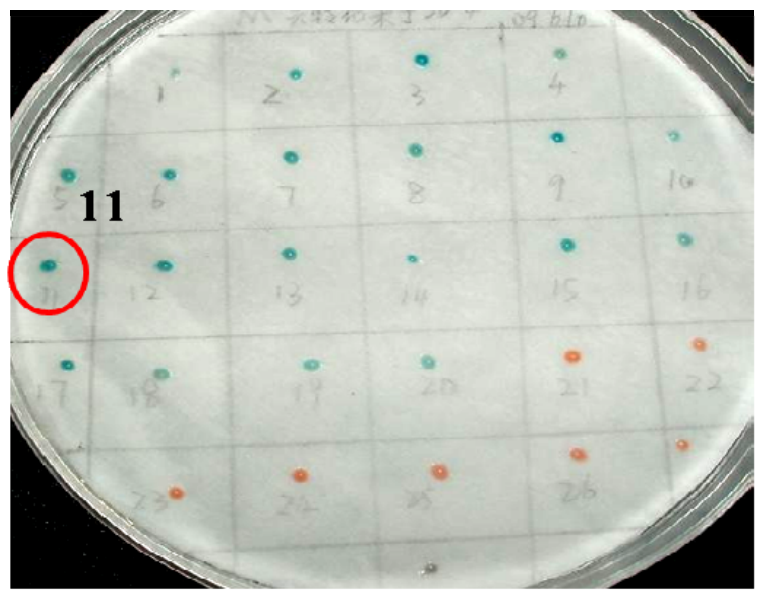

C

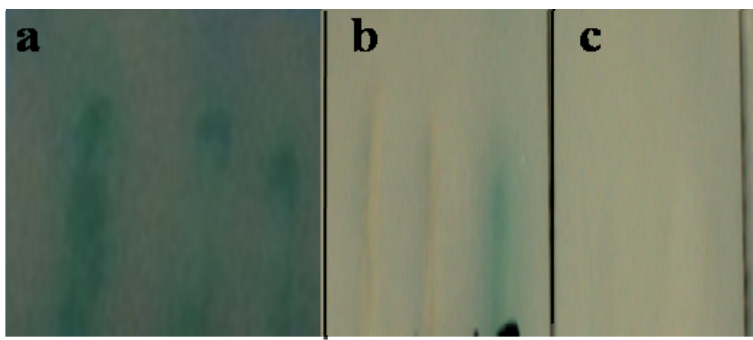

B

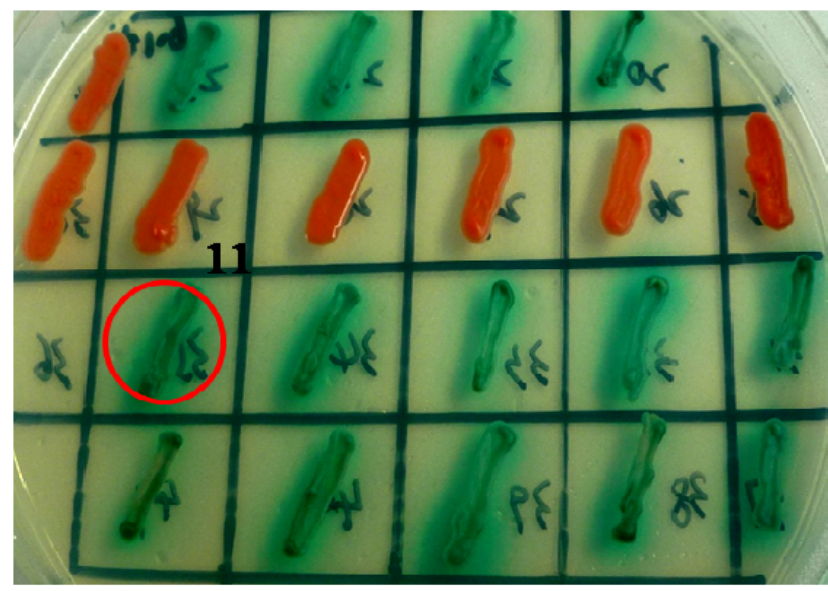

D

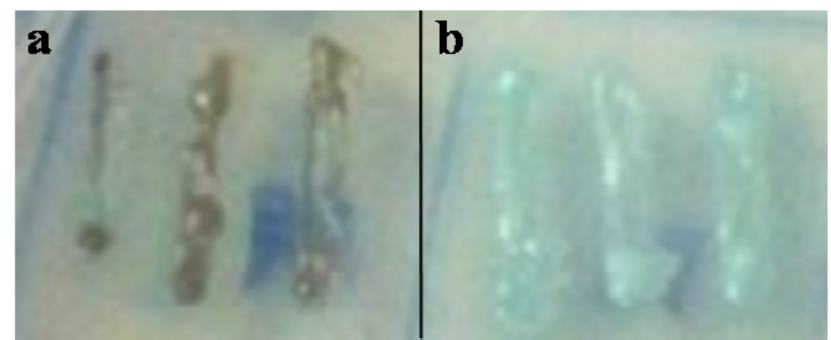


Figure 2. Fluorescence resonance energy transfer (FRET) analysis of the interaction between SelM' and Gal-1. (A) Representative laser scanning microscopy images of HeLa cells cotransfected empty plasmids pECFP-C1 and pEYFP-C1 (negative control). (a) The fluorescence image of donor (CFP) before bleaching; (b) The fluorescence image of donor after bleaching; (c) Diagram of the distance between donor (CFP) and receptor (YFP); (d) Donor fluorescence increment before and after bleaching; (e) FRET efficiency diagram; (f) Photobleaching curve (solid line for donor fluorescence and dashed line for receptor fluorescence); (B) Cells co-transfected pECFP-C1-SelM' and pEYFP-C1-Gal-1. (a) The fluorescence image of donor (CFP-SelM') before bleaching; (b) The fluorescence image of donor after bleaching; (c) Diagram of the distance between donor (CFP-SelM') and receptor (YFP-Gal-1); (d) Donor fluorescence increment before and after bleaching; (e) FRET efficiency diagram; (f) Photobleaching curve (solid line for donor fluorescence and dashed line for receptor fluorescence).

$\mathbf{A}$
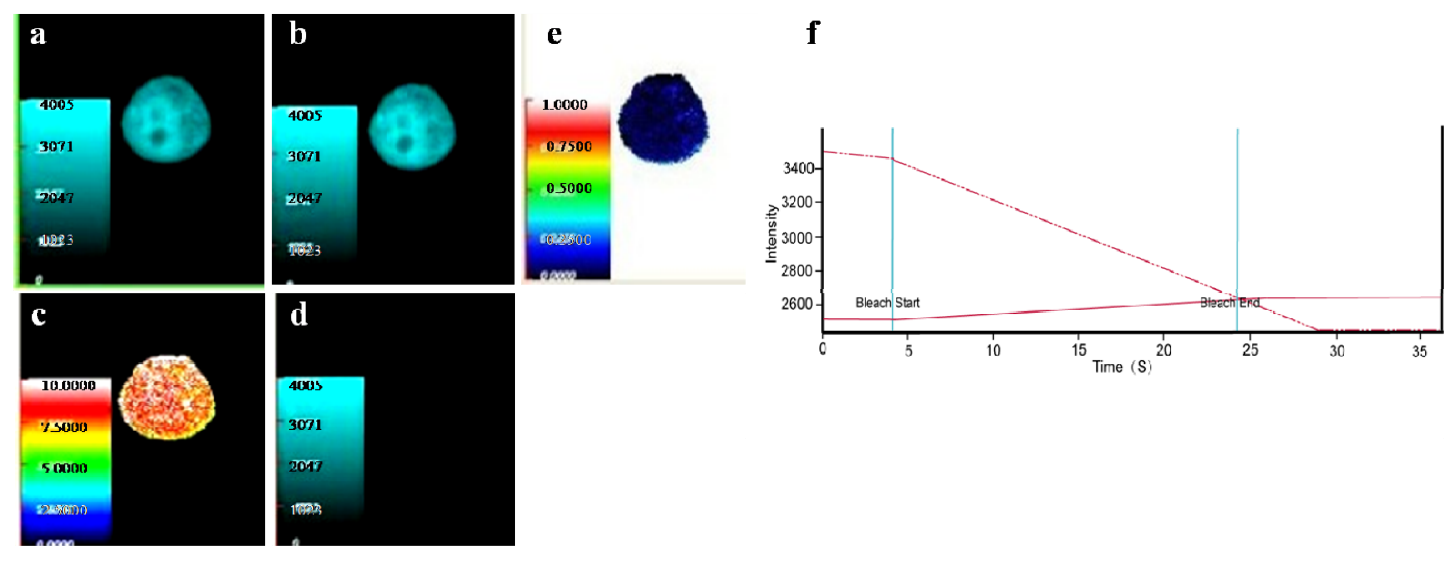

$\mathbf{B}$
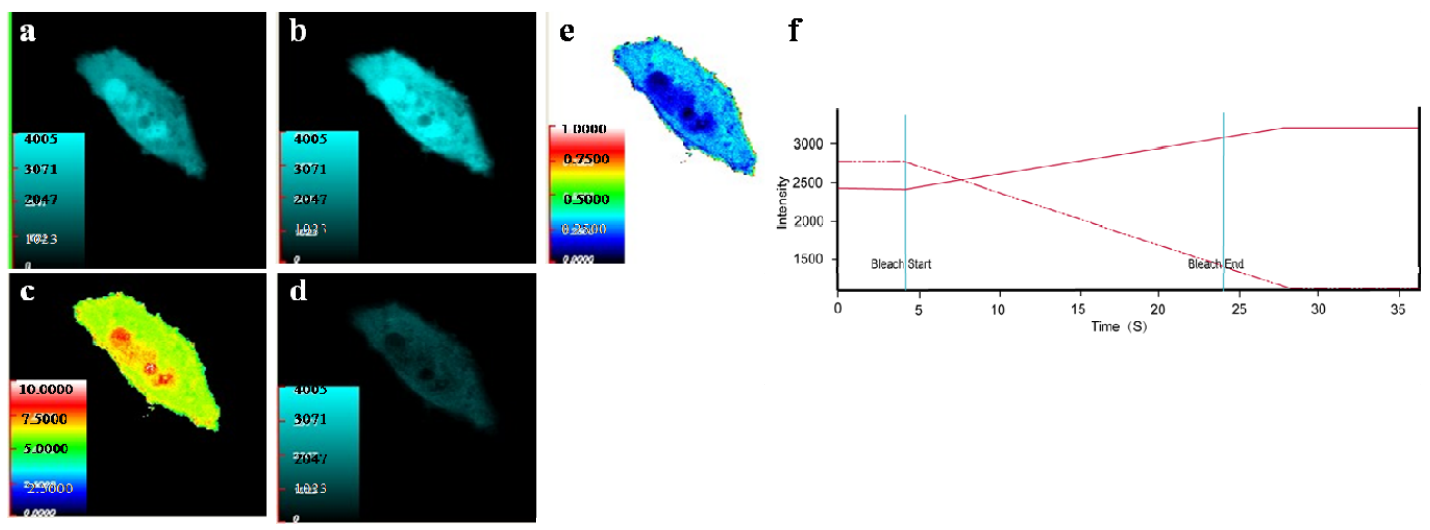

\subsection{Verification of the Direct Protein Interaction by GST Pull-Down Assay}

The open reading frame of Gal-1 was inserted into the GST expression vector pGEX-5X-1. The fusion protein GST-Gal-1 was expressed in E. coli and analyzed by Western blot (WB) as shown in Figure 3A. The PVDF membrane was treated with the enhanced chemiluminescent assay to reveal GST-Gal-1 (lane 1) and GST (lane 2) using GST antibody. As shown in Figure 3B, purified SelM' with His tag was bound to the GST-Gal-1-binding beads (lane 1) but not the GST-binding beads (lane 2) using anti-His antibody, where purified SelM' with His tag was used as a positive control to ascertain the 
reliability of WB analysis (lane 3). Those GST pull-down results verified the direct interaction between Gal-1 and SelM' in vitro.

Figure 3. GST pull-down analysis of the interaction between His-tagged SelM' and GST-Gal-1. (A) Western blot (WB) analysis of the expression of GST and GST-Gal-1 with GST antibody. Lane 1, GST-Gal-1 (42 kDa); lane 2, GST (27 kDa); (B) GST pull-down analysis of the protein interaction with His antibody. Equal amount of GST-Gal-1 (lane 1) or GST (lane 2) was bound to glutathione-Sepharose beads. After washing, His-tagged SelM' bound to GST-Gal-1 was eluted and analyzed by WB with anti-His antibody (lane $\mathbf{1}$ ). Purified SelM' protein with His tag was used as a positive control (lane 3).

A

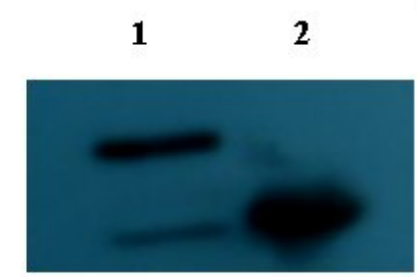

$\mathbf{B}$

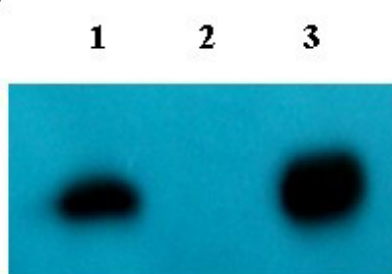

\subsection{Verification of the Protein Interaction by Co-Immunoprecipitation Assay}

Two plasmids pCMV-HA-Gal-1 and pCMV-Myc-SelM' were constructed and cotransfected into HEK293T cells. Mouse monoclonal c-Myc antibody was used for immunoprecipitation (IP), and monoclonal HA antibody was used for WB. Co-IP results (Figure 4) showed a $\sim 18 \mathrm{kDa}$ protein corresponding to Gal-1 in the cells cotransfected with pCMV-HA-Gal-1 and pCMV-Myc-SelM' (lane 2), but not in the negative control cells co-transfected with pCMV-Myc and pCMV-HA-Gal-1 (lane 3). Meanwhile, WB analysis was performed for the cells cotransfected with pCMV-HA-Gal-1 and pCMV-Myc-SelM' (lane 1) and the control cells co-transfected with pCMV-Myc and pCMV-HA-Gal-1 (lane 4). Those results further confirm the interaction between Gal-1 and SelM' in mammalian cells.

Figure 4. Co-immunoprecipitation analysis of the interaction between Myc-tagged SelM' and HA-tagged Gal-1. Lanes 1 and 2, HEK293T cells were cotransfected plasmids pCMV-Myc-SelM' and pCMV-HA-Gal-1. The cell lysates were analyzed either directly by WB with HA antibody (lane 1, as a positive control), or by IP with C-Myc antibody and WB with HA antibody (lane 2). Lanes 3 and 4, cells were cotransfected plasmids pCMV-Myc and pCMV-HA-Gal-1. The cell lysates were analyzed either by immunoprecipitation (IP) with $C$-Myc antibody and WB with HA antibody (lane 3), or directly by WB with HA antibody as a positive control (lane 4).

IP : anti- Myc

WB : anti-HA

$\begin{array}{llll}- & + & + & - \\ 1 & 2 & 3 & 4\end{array}$

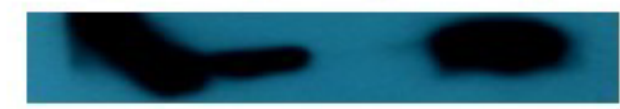




\section{Discussion}

SelM is located inside cells. The $N$-terminal signal peptide within the SelM sequence guides SelM to perinuclear structures, including ER and Golgi [10], rather than secreting it into extracellular media. It has been observed that, when the $N$-terminal signal peptide was present, SelM was expressed in ER, whereas no specific localization was seen for SelM in the absence of the signal peptide [10]. In our experiments, the $\mathrm{N}$-terminal signal peptide was cut off in order to use the yeast two-hybrid system for screening the human fetal cDNA library. The $N$-terminal-cutoff SelM was also observed to be distributed nonspecifically in the cytoplasm.

It is well known that selenoproteins, including SelM, are difficult to be overexpressed due to the presence of Sec encoded by TGA, a traditional stop codon. Thus, the structure of SelM was analyzed by the mutation of Sec to Cys [15], which did not change the configuration or active-site of SelM. The structure of SelM contains a thioredoxin-like domain and an active-site redox motif arranged in the form of CXXU [15]. The thioredoxin-like domain is composed of a mixed four-stranded $\beta$-sheet and three interspersed $\alpha$-helices. The active-site redox motif of SelM is located between the $\mathrm{C}$ terminus of strand $\beta 1$ and the $N$ terminus of helix $\alpha 1$ of the thioredoxin-like doman. Both active-site redox residues (Cys and Sec) are surface accessible. The CXXU motif of SelM is capable of forming a reversible mixed selenenylsulfide bond during the catalytic cycle of oxidation and reduction. Reduction of the active-site selenenylsulfide bond results in localized conformational changes that are centered on the redox motif. Due to the special structure of SelM, the Sec residue in SelM always functions with the adjacent Cys, similar to other protein disulfide isomerases with the redox active site of the CXXC motif. Thus, mutation of Sec to Cys in SelM does not change the structure or function of the active-site redox motif, and it should not produce a significant difference in the interaction between SelM and Gal-1.

Gal-1, an endogenous mammalian lectin, is a $14.5-\mathrm{kDa}$ soluble protein present both inside and outside cells, and has both intracellular and extracellular functions [16-19]. The extracellular functions require the carbohydrate-binding properties of dimeric Gal-1 while the intracellular ones are associated with carbohydrate-independent interactions between Gal-1 and other proteins [19]. Human lung fibroblast cells contained high concentrations of intracellular Gal-1, whose characteristics in structure and activity decided its intracellular localization. The thiol groups of Gal-1 in reduced form limited the protein to be located intracellularly [20]. Meanwhile, no signal peptide sequence was found before the $N$-terminal alanine to direct the protein for secretion. The $N$-terminal alanine is blocked with an acetyl group, and the acetylated $N$-terminal amino groups are usually associated with cytoplasmic proteins $[19,20]$. Interestingly, in most cases the lectin activity of Gal-1 is observed when it is extracellular, while the protein-protein interactions of Gal-1 concern its intracellular functions. The proteins that have been thus far identified which interact in a carbohydrate-independent manner with Gal-1 are not structurally related to each other and do not seem to share any common domains or motifs [19]. The galectin sites that are involved in these interactions have not yet been established. Thus, it is difficult to predict the interacting domains between Gal-1 and SelM, and this work is worth investigating further.

Gal-1 is widely expressed in mouse brain neurons, neural stem cells, and neuroblasts [21,22]. It regulates neural cell fates, such as cell proliferation, differentiation, and death [23-26]. It is essential for 
generating new neurons and in the recovery from brain damage [17,27]. Gal-1 also plays important roles in adult neural stem cells (NSCs) under both physiological and pathological conditions [28]. Gal-1 deficiency resulted in attenuated proliferation of neural progenitors in the hippocampal dentate gyrus (DG). Astrocytes promote neurogenesis in the adult hippocampus [29] and Gal-1 expressed in activated astrocytes is involved in the promotion of insult-induced neurogenesis in the DG [27]. Gal-1 also induces brain-derived neurotrophic factor (BDNF) in astrocytes to promote adult neurogenesis [30-32]. In addition, Gal-1 is a downstream target upregulated by $\Delta \mathrm{FosB}$, a protein induced immediately after ischemia in the DG of hippocampus [23,33], especially in neurons resistant to the injury. It is widely accepted that activated microglia exert dual functions, that is, pro-inflammatory (M1) and anti-inflammatory (M2) [34]. M1 microglia plays a key role in neuronal degeneration [35]. Gal-1 promoted the deactivation of M1 microglia and upregulated the activation of M2 microglia by modulating the signaling pathways of CREB, p38-MAPK, and NF- $\kappa \mathrm{B}$, which eventually prevented neurodegeneration and promoted neuroprotection [35,36]. Gal-1 may thus be considered as a means for the prevention of neuronal loss in cases of injury to the central nervous system [19,37]. SelM is an antioxidative protein that surpresses ROS level and protects neuronal cells [16]. Interestingly, SelM has also been reported to have neuroprotective function in previous reports [14]. In this study, SelM was found to interact with Gal-1, suggesting that the neuroprotection of SelM may be carried out indirectly by Gal-1. A detailed mechanism remains to be investigated in future studies.

\section{Experimental Section}

\subsection{Materials and Reagents}

The yeast strains Y2HGold and AH109, human fetal brain cDNA library and plasmids pGADT7, pGBKT7-53, pGBKT7-Lam, and pGADT7-T were purchased from Clontech (Mountainview, CA, USA). NpGBKT7 was kindly provided by Professor Keke Huo in the Fudan University. The E. coli strain TOP10 was stored in our lab. Myc, HA, His, and GST monoclonal antibodies, HRP-goat anti-mouse IgG and Protein-G agarose were purchased from SANTA CRUZ (Santa Cruz, Dallas, TX, USA). DNA-MATE for transfecion was from Bonataike (Shenzhen, China). The pECFP-C1 and pEYFP-C1 vectors were gifted by Professor Shengli Tian in the Shenzhen University.

\subsection{Gene Amplification and Plasmid Construction}

Full-length SelM gene was PCR-amplified from the human fetal brain cDNA library. The $N$-terminal of SelM containing 27 amino acids was predicted online to be a transmembrane region, and it was cut off to let SelM possible for yeast two-hybrid screening. Meanwhile, SelM was site-directedly mutated to SelM' by changing the in-frame Sec-coding TGA to the Cys-coding TGC in the primer, and amplifying the ORF of SelM via overlapping PCR. The primers were designed and shown in Table 1. The amplified $S e l M^{\prime}$ fragment was used to construct the bait plasmid $\mathrm{NpGBKT}_{7}-$ SelM' $^{\prime}$ for the following yeast two-hybrid assay. 
Table 1. The primer sequences used for PCR amplification of SelM and SelM'.

\begin{tabular}{|c|c|}
\hline Name of DNA fragment & PCR primer sequence $5^{\prime} \rightarrow 3^{\prime}$ \\
\hline \multirow{2}{*}{ The ORF of SelM } & F:5'-TTGGCCATTACGGCCATGAGCCTCCTGTTGCCTCCGCTG-3' \\
\hline & R:5'-TTGGCCGAGGCGGCCCTACAGGTCAGCGTGGTCCGAAGT-3' \\
\hline \multirow{2}{*}{ The upstream part of SelM' } & 'GCCTCCTGTTGCCTCCGCTG-3' \\
\hline & R:5'-TTGGCCGAGGCGGCCTAGGCGGTTCAGCTG GCATCCCCC-3' \\
\hline \multirow{2}{*}{$\begin{array}{l}\text { The downstream part of } \\
\text { SelM' }\end{array}$} & 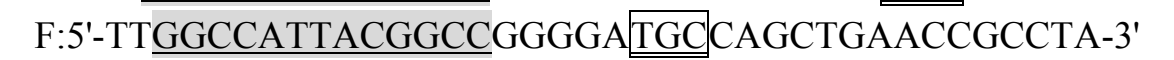 \\
\hline & R:5'-TTGGCCGAGGCGGCCCTACAGGTCAGCGTGGTCCGAAGT-3' \\
\hline \multirow{2}{*}{ The truncated SelM } & F:5'-TTGGCCATTACGGCCATGCGGCCGGACTGGAACCGTCTGA-3' \\
\hline & R:5'-TTGGCCGAGGCGGCCCTACAGGTCAGCGTGGTCCGAAGTT-3' \\
\hline
\end{tabular}

F: forward primer; R: reverse primer.

\subsection{Library Screening via the Yeast Two-Hybrid System}

Yeast transformation and two-hybrid screening were performed using the bait plasmid $\mathrm{NpGBKT}_{7}-\mathrm{SelM}^{\prime}$ to screen the human fetal brain cDNA library, following the procedures described in the Yeastmaker ${ }^{\mathrm{TM}}$ Yeast Transformation System 2 User Manual and the MatchmakerTM GAL4 Two-Hybrid System 3 \& Libraries User Manual (Clontech, Mountain View, CA, USA).

\subsection{Mammalian Cell Culture}

Human embryonic kidney 293T cells (HEK293T) and Hela cells were cultured in Dulbecco's Modified Eagle's Medium (DMEM) supplemented with 10\% $(v / v)$ fetal bovine serum and maintained at $37^{\circ} \mathrm{C}$ in $5 \% \mathrm{CO}_{2}$.

\subsection{Fluorescence Resonance Energy Transfer (FRET) Detection}

The FRET method of acceptor photobleaching was performed to validate the interacting proteins. Plasmids pECFP-C1-SelM' and pEYFP-C1-Gal-1 were constructed and sequence-verified. Hela cells were cotransfected pECFP-C1-SelM' and pEYFP-C1-Gal-1 for FRET analysis using laser confocal microscope (OLYMPUS, FV1000, Tokyo, Japan), while plasmids pECFP-C1 and pEYFP-C1 were cotransformed into the cells as the negative control. The method of acceptor photobleaching was performed as described previously [38,39].

\subsection{GST Pull-Down Assay and Western Blot Analysis}

His-tagged SelM' protein was overexpressed and purified in our lab [40,41]. The ORF of Gal-1 was cloned from the human fetal brain cDNA library and inserted into the GST expression vector of pGEX-5X-1. Gal-1 protein was expressed in the E. coli BL21 (DE3) pLysS cells. Glutathione (GSH)-Sepharose 4B beads were respectively pretreated with the GST or GST-Gal-1 bacterial cell lysate before binding with GST or GST-Gal-1. Approximately $1 \mathrm{mg}$ of GST or GST-Gal-1 was adsorbed to $20 \mu \mathrm{L}$ of the pretreated beads. His-tagged SelM' was incubated with the GST/GST-Gal-1 treated beads or non-treated beads (as a control) at $4{ }^{\circ} \mathrm{C}$ overnight in the NETN buffer $(50 \mathrm{mM}$ Tris-HCl, pH 7.5, $200 \mathrm{mM} \mathrm{NaCl}, 2 \mathrm{mM}$ EDTA, 0.1\% NP-40, with addition of $5 \mu \mathrm{L} 10 \mathrm{mg} / \mathrm{mL}$ PMSF before used). After extensive wash with phosphate buffer saline (PBS), the beads were suspended in 
$2 \times$ protein loading buffer, boiled for $5 \mathrm{~min}$, centrifuged at 13,000 rpm, and subjected to SDS-PAGE. Proteins separated on the gel were transferred onto a PVDF membrane. The membrane was immersed in the blocking buffer overnight. The blots were incubated with polyclonal His antibody $(1: 1000)$ in TBST (50 mM Tris-HCl, pH 7.4, $150 \mathrm{mM} \mathrm{NaCl,} \mathrm{0.1 \%} \mathrm{Tween} \mathrm{20)} \mathrm{containing} \mathrm{5 \%} \mathrm{non-fat} \mathrm{dried} \mathrm{milk} \mathrm{for}$ $2 \mathrm{~h}$ at room temperature. The membranes were then washed with TBST three times and incubated with HRP-labeled secondary antibodies $(1: 10,000)$ for $1 \mathrm{~h}$. Detection of HRP was performed using chemiluminescent substrate from Bonataike (Shenzhen, China).

\subsection{Co-Immunoprecipitation Assay}

HEK293T cells were cotransfected the plasmids pCMV-Myc-SelM' and pCMV-HA-Gal-1. Forty-eight hours after transfection, the cells were lysed in RIPA solution (Bonataike, Shenzhen, China). The lysates were centrifuged at $13,000 \mathrm{rpm}$ for $30 \mathrm{~min}$ at $4{ }^{\circ} \mathrm{C}$. The supernatants were collected and total protein amount was measured using the BCA method. For immunoprecipitation, the supernatant of cell lysate corresponding to $1 \mathrm{mg}$ of total protein was precleared by Protein G-agarose beads to minimize nonspecific binding. Then the precleared supernatnat was incubated with $2 \mu \mathrm{g}$ of anti-Myc antibody for $1 \mathrm{~h}$ at $4{ }^{\circ} \mathrm{C}$, followed by incubation with protein $\mathrm{G}$-agarose beads overnight at $4{ }^{\circ} \mathrm{C}$. The bound proteins were washed thrice with lysis buffer and dissociated with the beads via boiling and centrifugation. The collected proteins were suspended in $2 \times$ protein loading buffer, separated by SDS-PAGE and analyzed by Western blot using the primary antibody of anti-HA.

\section{Conclusions}

The interactive protein of SelM in the human brain was investigated in this paper. A human fetal brain cDNA library was screened by $S e l M^{\prime}$ using the yeast two-hybrid system. A new interactive protein of SelM' was screened out and sequence-analyzed to be Gal-1. Then, Gal-1 ORF was amplified from the fetal brain cDNA library and used for the construction of different plasmids. The interaction between SelM' and Gal-1 was further verified by the results of FRET, GST pull-down and co-IP. As Gal-1 plays an important role in neuroprotection, the discovery implies that SelM may implement neuroprotection through its interaction with Gal-1. The present paper provides a new direction for studying the biological function of SelM in the human brain.

\section{Acknowledgments}

This work was funded by the National Natural Science Foundation of China (No. 31070731, 21271131, 81372984) and by the Natural Science Foundation of Guangdong Province (No. 10151806001000023). We would like to thank Keke Huo in the College of Life Sciences, Fudan University, for his technique training of yeast-two hybridization.

\section{Conflicts of Interest}

The authors declare no conflict of interest. 


\section{References}

1. Godwin, K.; Kuchel, R.; Buckley, R. The effect of selenium on infertility in ewesgrazing improved pastures. Aust. J. Exp. Agric. Anim. Husb. 1970, 10, 672-678.

2. Paszkowski, T.; Traub, A.; Robinson, S.; McMaster, D. Selenium dependent glutathione peroxidase activity in human follicular fluid. Clin. Chim. Acta 1995, 236, 173-180.

3. Sies, H. Biochemistry of oxidative stress. Angew. Chem. Int. Ed. Engl. 1986, 25, 1058-1071.

4. Bellinger, F.P.; Raman, A.V.; Reeves, M.A.; Berry, M.J. Regulation and function of selenoproteins in human diseases. Biochem. J. 2009, 422, 11-22.

5. Koller, L.D.; Exon, J.H. The two faces of selenium deficiency and toxicity-Are similar in animals and man. Can. J. Vet. Res. 1986, 50, 297-306.

6. Rayman, M.P. The importance of selenium to human health. Lancet 2000, 356, 233-241.

7. Berry, M.J.; Tujebajeva, R.M.; Copeland, P.R.; Xu, X.M.; Carlson, B.A.; Martin, G.W., III; Low, S.C.; Mansell, J.B.; Grundner-Culemann, E.; Harney, J.W.; et al. Selenocysteine incorporation directed from the 3'UTR: Characterization of eukaryotic EFsec and mechanistic implications. Biofactors 2001, 14, 17-24.

8. Gromer, S.; Eubel, J.K.; Jacob, J. Human selenoproteins at a glance. Cell Mol. Life Sci. 2005, 62, 2414-2437.

9. Kryukov, G.V.; Castellano, S.; Novoselov, S.V.; Lobanov, A.V.; Zehtab, O.; Guigó, R.; Gladyshev, V.N. Characterization of mammalian selenoproteomes. Science 2003, 300, 1439-1443.

10. Korotkov, K.V.; Novoselov, S.V.; Hatfield, D.L.; Gladyshev, V.N. Mammalian selenoprotein in which selenocysteine ( $\mathrm{Sec}$ ) incorporation is supported by a new form of sec insertion sequence element. Mol. Cell Biol. 2002, 22, 1402-1411.

11. Labunskyy, V.M.; Hatfield, D.L.; Gladyshev, V.N. The Sep15 protein family: Roles in disulfide bond formation and quality control in the endoplasmic reticulum. IUBMB Life 2007, 59, 1-5.

12. Hwang, D.Y.; Sin, J.S.; Kim, M.S.; Yim, S.Y.; Kim, Y.K.; Kim, C.K.; Kim, B.G.; Shim, S.B.; Jee, S.W.; Lee, S.H.; et al. Overexpression of human selenoprotein M differentially regulates the concentrations of antioxidants and $\mathrm{H}_{2} \mathrm{O}_{2}$, the activity of antioxidant enzymes, and the composition of white blood cells in a transgenic rat. Int. J. Mol. Med. 2008, 21, 169-179.

13. Yim, S.Y.; Chae, K.R.; Shim, S.B.; Hong, J.T.; Park, J.Y.; Lee, C.Y.; Son, H.J.; Sheen, Y.Y.; Hwang, D.Y. ERK activation induced by selenium treatment significantly downregulates beta/gamma-secretase activity and Tau phosphorylation in the transgenic rat overexpressing human selenoprotein M. Int. J. Mol. Med. 2009, 24, 91-96.

14. Reeves, M.A.; Bellinger, F.P.; Berry, M.J. The neuroprotective functions of selenoprotein M and its role in cytosolic calcium regulation. Antioxid. Redox. Signal 2010, 12, 809-818.

15. Ferguson, A.D.; Labunskyy, V.M.; Fomenko, D.E.; Araç, D.; Chelliah, Y.; Amezcua, C.A.; Rizo, J.; Gladyshev, V.N.; Deisenhofer, J. NMR structures of the selenoproteins Sep15 and SelM reveal redox activity of a new thioredoxin-like family. J. Biol. Chem. 2006, 281, 3536-3543.

16. Kato, T.; Ren, C.H.; Wada, M.; Kawanami, T. Galectin-1 as a potential therapeutic agent for amyotrophic lateral sclerosis. Curr. Drug Targets 2005, 6, 407-418. 
17. Ishibashi, S.; Kuroiwa, T.; Sakaguchi, M.; Sun, L.; Kadoya, T.; Okano, H.; Mizusawa, H. Galectin-1 regulates neurogenesis in the subventricular zone and promotes functional recovery after stroke. Exp. Neurol. 2007, 207, 302-313.

18. Gaudet, A.D.; Steeves, J.D.; Tetzlaff, W.; Ramer, M.S. Expression and functions of galectin-1 in sensory and motoneurons. Curr. Drug Targets 2005, 6, 419-425.

19. Camby, I.; Mercier, M.L.; Lefranc, F.; Kiss, R. Galectin-1: A small protein with major functions. Glycobiology 2006, 16, 137R-157R.

20. Clerch, L.B.; Whitney, P.; Hass, M.; Brew, K.; Miller, T.; Werner, R.; Massaro, D. Sequence of a full-length cDNA for rat lung beta-galactoside-binding protein: Primary and secondary structure of the lectin. Biochemistry 1988, 27, 692-629.

21. Sakaguchi, M.; Shingo, T.; Shimazaki, T.; Okano, H.J.; Shiwa, M.; Ishibashi, S.; Oguro, H.; Ninomiya, M.; Kadoya, T.; Horie, H.; et al. A carbohydrate-binding protein, galectin-1, promotes proliferation of adult neural stem cells. Proc. Natl Acad. Sci. USA 2006, 103, 7112-7117.

22. Imaizumi, Y.; Sakaguchi, M.; Morishita, T.; Ito, M.; Poirier, F.; Sawamoto, K.; Okano, H. Galectin-1 is expressed in early-type neural progenitor cells and down-regulates neurogenesis in the adult hippocampus. Mol. Brain 2011, 4, 7.

23. Kurushima, H.; Ohno, M.; Miura, T.; Nakamura, T.Y.; Horie, H.; Kadoya, T.; Ooboshi, H.; Kitazono, T.; Ibayashi, S.; Iida, M.; et al. Selective induction of DeltaFosB in the brain after transient forebrain ischemia accompanied by an increased expression of galectin-1, and the implication of DeltaFosB and galectin-1 in neuroprotection and neurogenesis. Cell Death Differ. 2005, 12, 1078-1096.

24. Tahara, K.; Tsuchimoto, D.; Tominaga, Y.; Asoh, S.; Ohta, S.; Kitagawa, M.; Horie, H.; Kadoya, T.; Nakabeppu, Y. DetaFosB, but not FosB, induces delayed apoptosis independent of cell proliferation in the Rat1 a embryo cell line. Cell Death Differ. 2003, 10, 496-507.

25. Miura, T.; Takahashi, M.; Horie, H.; Kurushima, H.; Tsuchimoto, D.; Sakumi, K.; Nakabeppu, Y. Galectin-1beta, a natural monomeric form of galectin-1 lacking its six amino-terminal residues promotes axonal regeneration but not cell death. Cell Death Differ. 2004, 11, 1076-1083.

26. Miura, T.; Ohnishi, Y.; Kurushima, H.; Horie, H.; Kadoya, T.; Nakabeppu, Y. Regulation of the neuronal fate by DeltaFosB and its downstream target, galectin-1. Curr. Drug Targets. 2005, 6, $437-444$.

27. Kajitani, K.; Nomaru, H.; Ifuku, M.; Yutsudo, N.; Dan, Y.; Miura, T.; Tsuchimoto, D.; Sakumi, K.; Kadoya, T.; Horie, H.; et al. Galectin-1 promotes basal and kainate-induced proliferation of neural progenitors in the dentate gyrus of adult mouse hippocampus. Cell Death Differ. 2009, 16, $417-427$.

28. Sakaguchi, M.; Okano, H. Neural stem cells, adult neurogenesis, and galectin-1: From bench to bedside. Dev. Neurobiol. 2012, 72, 1059-1067.

29. Song, H.; Stevens, C.F.; Gage, F.H. Astroglia induce neurogenesis from adult neural stem cells. Nature 2002, 417, 39-44.

30. Sasaki, T.; Hirabayashi, J.; Manya, H.; Kasai, K.; Endo, T. Galectin-1 induces astrocyte differentiation, which leads to production of brain-derived neurotrophic factor. Glycobiology 2004, $14,357-363$. 
31. Zhao, C.; Deng, W.; Gage, F.H. Mechanisms and functional implications of adult neurogenesis. Cell 2008, 132, 645-660.

32. Lee, J.; Duan, W.; Mattson, M.P. Evidence that brain-derived neurotrophic factor is required for basal neurogenesis and mediates, in part, the enhancement of neurogenesis by dietary restriction in the hippocampus of adult mice. J. Neurochem. 2002, 82, 1367-1375.

33. Nishioka, T.; Sakumi, K.; Miura, T.; Tahara, K.; Horie, H.; Kadoya, T.; Nakabeppu, Y. FosB gene products trigger cell proliferation and morphological alteration with an increased expression of a novel processed form of galectin-1 in the rat 3Y1 embryo cell line. J. Biochem. 2002, 131, 653-661.

34. Kobayashi, K.; Imagama, S.; Ohgomori, T.; Hirano, K.; Uchimura, K.; Sakamoto, K.; Hirakawa, A.; Takeuchi, H.; Suzumura, A.; Ishiguro, N.; et al. Minocycline selectively inhibits M1 polarization of microglia. Cell Death Dis. 2013, 4, e525.

35. Motohiro, N.; Minoru, F. Galectin-1 for neuroprotection? Immunity 2012, 37, 187-189.

36. Starossom, S.C.; Mascanfroni, I.D.; Imitola, J.; Cao, L.; Raddassi, K.; Hernandez, S.F.; Bassil, R.; Croci, D.O.; Cerliani, J.P.; Delacour, D.; et al. Galectin-1 deactivates classically activated microglia and protects from inflammation-induced neurodegeneration. Immunity 2012, 37, 249-263.

37. Egnaczyk, G.F.; Pomonis, J.D.; Schmidt, J.A.; Rogers, S.D.; Peters, C.; Ghilardi, J.R.; Mantyh, P.W.; Maggio, J.E. Proteomic analysis of the reactive phenotype of astrocytes following endothelin-1 exposure. Proteomics 2003, 3, 689-398.

38. Chen, P.; Wang, C.; Ma, X.; Zhang, Y.; Liu, Q.; Qiu, S.; Liu, Q.; Tian, J.; Ni, J. Direct interaction of selenoprotein $\mathrm{R}$ with clusterin and its possible role in Alzheimer's disease. PLoS One 2013, 8, e66384.

39. Liang, X.Y.; Liu, Q.; Chen, P.; Huo, K.K.; Hu, T.Y.; Ni, J.Z. Screen and verification of interactive protein of selenoprotein k in human liver. Chem. J. Chin. Univ. 2012, 23, 1-5.

40. Du, X.B.; Li, H.P.; Wang. Z.; Qiu, S.; Liu, Q.; Ni, J. Selenoprotein P and selenoprotein M block $\mathrm{Zn}^{2+}$-mediated $\mathrm{A} \beta_{42}$ aggregation and toxicity. Metallomics 2013, 5, 861-870.

41. Chen, P.; Wang, R.D.; Ma, X.J.; Liu, Q.; Ni, J.Z. Different forms of selenoprotein M differentially affect A $\beta$ aggregation and ROS generation. Int. J. Mol. Sci. 2013, 14, 4385-4399.

(C) 2013 by the authors; licensee MDPI, Basel, Switzerland. This article is an open access article distributed under the terms and conditions of the Creative Commons Attribution license (http://creativecommons.org/licenses/by/3.0/). 